\title{
DEBATES
}

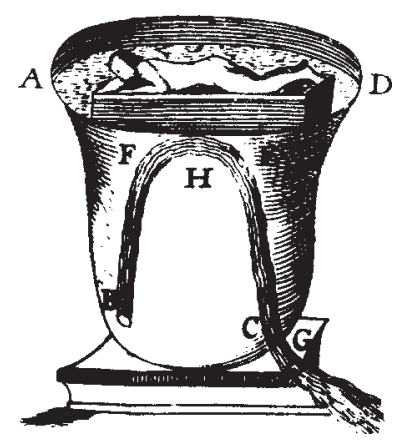

\section{CONTRA ALGUNAS CONCEPCIONES Y PREJUICIOS COMUNES DE LOS PROFESORES UNIVERSITARIOS DE CIENCIAS SOBRE LA DIDÁCTICA DE LAS CIENCIAS}

\author{
CAMPANARIO, JUAN MIGUEL \\ Aprendizaje de las Ciencias. Departamento de Física. Universidad de Alcalá de Henares. \\ 28871 Alcalá de Henares. Madrid \\ http://www.uah.es/otrosweb/jmc \\ juan.campanario@uah.es
}

Resumen. En este trabajo se analizan y cuestionan algunas ideas y concepciones sobre la didáctica de las ciencias comunes entre el profesorado universitario de ciencias.

Palabras clave. Enseñanza de las ciencias, concepciones docentes, profesores universitarios.

Summary. In this work I analyze and challenge some ideas and misconceptions on Science teaching that are common in university teachers of sciences.

Keywords. Science teaching, teaching beliefs, university teachers.

\section{INTRODUCCIÓN}

En los últimos años se ha prestado una atención cada vez mayor a la influencia del pensamiento del profesor en su trabajo, es decir, a cómo las ideas, concepciones y valores del docente influyen en sus estrategias de ense- 
ñanza y en su actuación en clase. Nos referimos a aspectos distintos del conocimiento de la propia asignatura o disciplina, como son las concepiones sobre la ciencia, la enseñanza, el aprendizaje, las motivaciones de los alumnos, los métodos docentes y otros aspectos relacionados con su actividad. Las investigaciones en esta área se han realizado utilizando metodologías diversas (Porlán, Rivero y Martín, 1997a) y existe un cuerpo cada vez más amplio de conocimientos acerca de las ideas y concepciones del profesor en ejercicio (Porlán, Rivero, Martín, 1997b) o en formación (Mellado, 1996; Campanario, 1998).

En particular, se sabe que los puntos de vista de los profesores sobre la enseñanza y el aprendizaje son quizá uno de los obstáculos más formidables que hay que vencer para conseguir un cambio en sus métodos de enseñanza. Como es sabido, en el caso de la universidad, predomina un monótono horizonte caracterizado, casi totalmente, por la dictadura propia de la clase magistral, a pesar de las propuestas que existen para utilizar otros enfoques (Campanario y Moya, 1999).

En este trabajo no vamos a abordar de manera sistemática las ideas y concepciones docentes de los profesores universitarios de ciencias. En vez de eso, se sigue un enfoque diferente: se seleccionan y analizan algunas de las concepciones inadecuadas y prejuicios más comunes de estos profesores sobre la didáctica de las ciencias y su papel en la enseñanza; se indaga en su posible origen y se proponen algunas reflexiones orientadas a poner en duda la validez de las mismas. Si las ideas se consideran inadecuadas, no es porque sean incorrectas, sino porque impiden o dificultan la toma de conciencia y de medidas para evitar los problemas que causan y las consecuencias que originan. Cabe realizar algunas precisiones:

1) No se pretende afirmar aquí que todos los profesores universitarios de ciencias estén de acuerdo con todas estas ideas.

2) No se trata tanto de rebatir las concepciones inadecuadas de los profesores, como de buscar las debilidades, inconsistencias o supuestos equivocados que las sustentan, así como, en algunos casos, los conflictos con otras pautas de razonamiento o actitudes más científicas.

3) Es posible que coexistan concepciones inconsistentes incluso en el mismo profesor. Ello se debe a que las ideas son implícitas (en muchos casos constituyen simplemente actitudes). Estas ideas pueden aflorar en contextos diferentes, lo cual da como resultado que no se detecte la contradicción.

Aunque se ha procurado agrupar las ideas y concepciones en unos bloques amplios más o menos relacionados entre sí, no se ha hecho un esfuerzo sistemático de recopilación de todas las concepciones identificadas en la investigación desarrollada en este terreno. Más bien, las concepciones comunes que aquí se analizan se han detectado (y discutido) durante la interacción con profesores universitarios de ciencias en nuestro trabajo diario en un entorno en el que estas ideas están ampliamente extendidas y firmemente arraigadas.

No somos excesivamente optimistas sobre el resultado de nuestro intento. Incluso, aunque las ideas y concepciones que analizamos se cuestionasen en algún tipo de curso o programa de formación de profesores universitarios, los resultados serían probablemente escasos. Las razones son diversas.

1) Muchas de las ideas que analizamos en este trabajo son de sentido común, lo que hace que su cuestionamiento pueda considerarse innecesario ( si no está roto, no lo arregles).

2) Por otra parte, sabemos que no basta con presentar evidencias en contra de las ideas propias para que éstas cambien. Incluso en la propia ciencia las teorías en conflicto con los datos pueden sobrevivir durante mucho tiempo. Es difícil cambiar una forma de entender una realidad determinada (una teoría) si no se sustituye por otra forma de entender dicha realidad (otra teoría).

3) Cuando en la forma propia de entender la realidad están implicados valores y puntos de vista, las dificultades aumentan, y mucho más si el cuestionamiento de las ideas y concepciones se confunde con el cuestionamiento de la eficacia profesional.

4) La arraigada creencia de que cualquiera que tenga un título de licenciado (o doctor) está técnicamente cualificado para dar clase en la universidad porque está legalmente reconocido para ello es difícil de combatir sin suscitar airadas reacciones. El cualquiera sirve que subyace tras esta concepción, está estrechamente relacionado con el no se necesita saber más, que acaba siendo la argumentación más común que escuchamos cuando planteamos la necesidad de una formación adicional psicopedagógica en el profesorado universitario (Campanario, 2002a).

En cualquier caso, creo que ponerse en el lugar de aquél al que tratamos de ayudar constituye un excelente ejercicio de empatía. Tratar de analizar la lógica que subyace en las concepciones docentes de los profesores universitarios es quizá el primer paso antes de abordar con éxito cualquier intento de formación didáctica. Además, este ejercicio nos ayuda a cuestionar y analizar las bases de nuestra área de conocimientos, muy proclive a las propuestas que hacen proclamaciones generalizadas de éxito en todas las circunstancias y contextos.

\section{PUNTOS DE VISTA GENERALES SOBRE LA EDUCACION Y LA ENSENANZA}

En este apartado abordamos opiniones generales sobre la enseñanza y la educación que tienen su base en percepciones personales de los profesores y, en muchos casos, en la necesidad de explicar la situación actual de la enseñanza de una manera razonable y tolerable. 


\section{La enseñanza actual es peor que la enseñanza de antes}

Una queja común entre los profesores universitarios es que la situación de la enseñanza antes era mejor. Los síntomas que más se denuncian son:

1) La peligrosa disminución de la calidad de la enseñanza secundaria (que, para muchos, sólo se concibe como una base necesaria para la enseñanza universitaria).

2) El descenso del nivel de la enseñanza en la universidad, que, según se dice, también ha bajado muchísimo: los alumnos no estudian, no están motivados, no quieren aprender, etc. Parece como si los nuevos tiempos hubiesen introducido un empeoramiento del sistema educativo.

Sin entrar a dilucidar si tales quejas se corresponden o no a la realidad, vale la pena citar aquí algunos elementos que sistemáticamente se olvidan cuando se hacen tales comparaciones:

1) El número de alumnos en el sistema educativo ha crecido considerablemente en los últimos decenios. También se ha prolongado la educación obligatoria. La educación universitaria es masiva y, de momento, masificada, si bien es cierto que están empezando a cambiar las cosas (y, claro, ahora las quejas son por la disminución del número de alumnos).

2) Los conocimientos científicos han experimentado en las últimas décadas una explosión sin precedentes. Los alumnos tienen que aprender más que antes y en menos tiempo que antes.

3) Los valores sociales han cambiado y ahora nos parecen buenas y aceptables cosas que antes estaban mal vistas, y viceversa. La ética del trabajo ha cambiado y esos cambios se reflejan en la educación.

Lo cierto es que las quejas relativas a la mala calidad de la enseñanza no son de ahora. Según Gibss y Fox, son más de 4.000 los artículos y libros publicados en los que docentes y académicos denuncian crisis escolares diversas (Gibss y Fox, 2000). Desde que en 1957 el lanzamiento del Sputnik por los soviéticos desencadenara la primera gran crisis de confianza en el sistema educativo americano, respetables profesores y sesudos académicos vienen formulando pesimistas predicciones acerca de la inminente catástrofe en los Estados Unidos derivada de la mala calidad en la enseñanza. El hecho de que ese país siga ocupando una posición política, militar, científica y tecnológica preponderante no es óbice, valladar o cortapisa para que se sigan generando predicciones apocalípticas allí. En nuestro país los lamentos que se lanzan desde las tribunas de prensa y las tertulias radiofónicas son similares.

Con todo lo anterior no queremos concluir que la situación actual sea buena o que estén equivocados los que se quejan de la mala calidad de la enseñanza. Simplemente queremos dejar constancia de que estos lamentos no son ni nuevos ni originales: haga memoria el lector y recuer- de las ténebres admoniciones y dolorosos lamentos al respecto de los cátedros de antaño. Tiempos atrás, los profesores también se quejaban de que la enseñanza era mejor antes.

\section{Las causas del mal funcionamiento del sistema educativo}

Una fuente inagotable de debates en el contexto universitario tiene que ver con las causas del funcionamiento defectuoso que se aprecia en el sistema educativo en todos sus niveles. Es posible que el lector mismo haya participado más de una vez en estos debates en los que suele haber una cierta unanimidad. Sin embargo, una actividad interesante consiste en hacer una recopilación de las causas por las cuales el sistema educativo funciona mal y clasificar estas causas de acuerdo con las siguientes dimensiones:

- Internas / externas al profesor.

- Controlables / incontrolables por el profesor.

- Estables / variables.

Hemos propuesto y realizado esta actividad en diversos contextos y siempre o casi siempre se llega a la misma conclusión: el profesor está inerme (o inane) y poco o nada tiene que ver con o puede hacer para remediar el desastre que denuncia. Parece como si la situación de la enseñanza poco tuviese que ver con él. El caso de la enseñanza secundaria es paradigmático: una de las quejas frecuentes antes era que los profesores tenían siempre escaso margen de decisión en la organización general de la enseñanza. Poco, entonces, podían hacer. Cuando, tras la última (y criticada) reforma se transfirió una gran parte de la responsabilidad del diseño de proyectos curriculares al centro escolar y al profesor, las quejas siguieron, esta vez en sentido contrario.

\section{EL PAPEL DE LA DIDÁCTICA DE LAS CIENCIAS EN LA ENSENANZA}

No puede decirse que las áreas de investigación relacionadas con la didáctica de las ciencias figuren entre las mejor consideradas por los profesores universitarios de ciencias. Las quejas y acusaciones que se formulan contra nosotros son diversas. A continuación se analizan algunas de las más comunes.

\section{La didáctica sólo busca el bien del estudiante en detrimento del profesor}

Una parte de las actitudes negativas hacia la didáctica tiene que ver con el supuesto papel de ésta como valedora del alumno. Parece, según una creencia muy extendida, que los que nos dedicamos a la didáctica de las ciencias tomamos a nuestro cargo la tarea de ser abogados defensores de oficio del estudiante cuando señalamos, por ejemplo, las carencias en la formación psicope- 
dagógica de los profesores y describimos la monotonía de sus estrategias docentes. El contexto general en la enseñanza que hace inevitable un cierto nivel de conflicto en las relaciones entre profesores y estudiantes hace el resto: todo el que no está absoluta y completamente a favor del profesor parece que está a favor del alumno.

Lo cierto es que una de las líneas de investigación en didáctica de las ciencias tiene que ver precisamente con los problemas de aprendizaje de las ciencias. Es natural que la investigación haya confirmado que la enseñanza tradicional resulte ineficaz en muchos casos para promover el aprendizaje significativo en los alumnos. La confusión frecuente entre la función formativa de la enseñanza y la función selectiva que suele desarrollarse en paralelo hace que sean comunes ideas tan extendidas como la que afirma que el buen alumno universitario aprenderá y aprobará en cualquier condición y contexto. Independientemente de que la afirmación anterior sea o no contrastable (se define buen alumno como aquél que sobrevive y sobrevive aquél que es buen alumno), lo cierto es que el estudiante medio (no confundir con mediocre) seguramente se beneficiará de que los profesores utilicen métodos docentes menos pasivos que los tradicionales. Conseguir que esta idea penetre en el sistema de pensamiento de la mayoría de los profesores universitarios de ciencias sin que se vean amenazados es todo un reto (Campanario, 2002a).

\section{La didáctica es innecesaria o perjudicial}

Uno de los argumentos que más se oye contra la necesidad de una formación didáctica del profesorado de ciencias de universidad es que dicha formación resulta innecesaria (Campanario, 2002a). Se argumenta, no sin cierta lógica, que siempre ha habido físicos y químicos sin que se supiera nada de psicología y didáctica y siempre los habrá, aunque no se apliquen métodos didácticos de enseñanza. Está muy extendida una vaga creencia según la cual el buen docente nace, no se hace. Las continuas referencias al carácter vocacional de nuestro trabajo parecen excluir la necesidad y aun la conveniencia de cualquier formación didáctica. Lo extendido de esta creencia se ilustra, por ejemplo, en las típicas películas sobre profesores en las que el buen docente desempeña bien su trabajo, debido, esencialmente, a sus características personales y, lógicamente, sin la intervención de ningún proceso de formación. Peor aún, otra de las acusaciones comunes que se hace contra los expertos en didáctica es que, si se siguieran sus recomendaciones y propuestas, la situación de la enseñanza sería todavía peor de lo que es en la actualidad.

Una prueba común que suele esgrimirse para reforzar esta acusación es el estado lamentable de la enseñanza secundaria, cuyo diseño actual se atribuye (erróneamente) a psicólogos, pedagogos y demás ralea. Se suele decir que la reforma de la enseñanza secundaria y las posteriores quejas y propuestas de contrarreforma demuestran que la didáctica puede complicar y empeorar las cosas que siempre se han hecho bien siguiendo el sentido común. Ante este razonamiento, de poco sirve que se analicen las frecuentes quejas y lamentos que se oyen sobre los actuales planes de estudio en la universidad y el escaso o nulo papel que en la reforma de dichos planes tuvieron los psicólogos, pedagogos y expertos en didáctica de las ciencias.

El tipo de crítica que abordamos en este apartado, se dirige con frecuencia a quienes mantenemos la necesidad de una formación psicopedagógica del profesorado de ciencias. Según muchos, la formación didáctica tiene sentido, fundamentalmente, en profesores de enseñanza primaria y (menos) en los de secundaria. Desde luego -se argumenta-, los profesores de la universidad no necesitan este tipo de formación porque se espera de los alumnos una madurez tal que puedan aprender casi con cualquier sistema (en realidad, con la hiperdominante clase magistral). Ciertamente, resulta difícil encontrar otros ejemplos de actividades profesionales (distintas de la enseñanza) en las que se recomiende a cualquiera que siga siempre las propias intuiciones o experiencias antes que las orientaciones derivadas de un proceso de formación (Campanario, 2002a).

Hay que reconocer que la afirmación que encabeza este apartado tiene su origen en actitudes y valores firmemente arraigados en el profesorado de ciencias. Estas actitudes y valores se han ido formando durante los largos años que los profesores han pasado en el sistema universitario aprendiendo una disciplina científica (y, de paso, implícitamente, aprendiendo una forma de enseñarla). Como tales actitudes es difícil cambiarlas simplemente saliendo al paso de ellas o proporcionando información, tal vez el camino más seguro para conseguir un cambio (aunque sea mínimo) consista en convencer al profesor de las ventajas que puede tener para el mismo conseguir una formación psicopedagógica.

\section{Las cuestiones relacionadas con la didáctica son opinables}

Debido a que la organización de las tareas de enseñanza es un tema en el que se admite y defiende una amplia libertad por parte del profesor (véase más adelante), no resulta raro que se piense que todas las cuestiones relacionadas con la didáctica son opinables de manera casi absoluta. Este autor ha sido testigo de animadas discusiones entre defensores de dos puntos de vista opuestos sobre la forma de hacer o enseñar algo en clase y de cómo prevalecía, al final, la opinión o la sensación de que los defensores de ambas posturas podían tener en buena parte razón simultáneamente (!). 
La actividad anterior debería bastar para entender que, al igual que en cualquier otra área, la contrastación experimental puede ser necesaria para decidir entre dos o más propuestas o alternativas (lo que invalida la supuesta opinabilidad absoluta de las cuestiones didácticas). Sin embargo, dicha contratación experimental debería tener en cuenta (entre otros), factores como:

1) La medida rigurosa y fiable del aprendizaje y de otros resultados de cualquier proceso de enseñanza (p.e., la motivación).

2) Los posibles factores o variables que contaminen la interpretación de los resultados.

3) La influencia de las expectativas de los investigadores en los resultados obtenidos y medidos.

El origen de la creencia en la opinabilidad absoluta tal vez esté en la diversidad de resultados que se pueden obtener en el contexto de clase cuando se realiza una intervención. Así, por ejemplo, es posible que dos profesores hagan (aparentemente) lo mismo en dos clases con alumnos (supuestamente) similares y obtengan resultados (aparentemente) diferentes. No cabe duda de que factores no controlados explican toda esta diversidad. La repetición de episodios de este tipo podría ayudarnos, tal vez, a entender el origen de la idea inadecuada tan extendida acerca de la opinabilidad absoluta de las cuestiones didácticas.

La supuesta opinabilidad absoluta se refleja, por ejemplo, en las primeras reacciones típicas ante un resultado de alguna investigación. Por ejemplo, ante una afirmación como «está comprobado que tanto los profesores como las profesoras asignan calificaciones más altas a un examen cuando creen que pertenece a un alumno que cuando creen que es de una alumna», las reacciones negativas más comunes son del tipo «no creo porque ...» o «eso conmigo no pasa porque...». En vez de solicitar información acerca de cómo se ha realizado el estudio (o la revisión y síntesis de estudios), de cómo se seleccionó la muestra de sujetos o cómo se han controlado variables que pudieran haber contaminado los datos, se cuestionan directamente las conclusiones «basándose en el sentido común» y en la experiencia propia. Es esta una manera de razonar que se aleja notablemente de la forma habitual de analizar y criticar los resultados de los experimentos y trabajos científicos que, por ejemplo, los propios profesores universitarios de ciencias quieren que sus alumnos desarrollen.

\section{La didáctica de las ciencias sólo es útil, válida, aceptable y tolerable si es aplicada o tiene aplicación inmediata para la preparación o desarrollo de las clases}

Los profesores de ciencias tienden a concebir la didáctica de las ciencias experimentales como una disciplina eminentemente aplicada. En consecuencia, se espera que los que trabajamos en esta área nos dediquemos a proponer nuevos métodos para que, si así lo estima oportuno, el profesor de ciencias los utilice y resuelva el problema de su clase diaria. En consecuencia, toda actividad que se aleje de este marco de actuación se estaría apartando del dominio de conocimientos y actuación propios de nuestra especialidad. Sin embargo, basta mirar cualquier revista del área (p.e., Enseñanza de las Ciencias) para descubrir que los especialistas en didáctica de las ciencias nos ocupamos también de problemas y temas tales como:

1)La historia, filosofía y epistemología de la ciencia como fundamentación del área y como elementos necesarios de la llamada alfabetización científica.

2)Los problemas cognitivos y motivacionales de aprendizaje de las ciencias, así como las actitudes de los alumnos (no sólo nos interesan los problemas de enseñanza).

3) Las ideas docentes de los profesores y su impacto en los métodos de enseñanza que éstos desarrollan.

4) Las interacciones ciencia-tecnología-sociedad.

Creer que la didáctica de las ciencias debe ocuparse únicamente de los problemas directos relacionados con la clase diaria es, salvando las distancias, como pensar que en física o química debe suprimirse cualquier investigación básica o teórica en beneficio de la aplicación inmediata al problema cotidiano de una industria determinada.

Por otra parte, la noción de que una disciplina, campo de investigación o área de conocimientos debe limitarse $a \ldots$ es, en sí misma, sorprendente y llamativa cuando tiene su origen en profesores universitarios (cuyo negocio consiste, precisamente, en transmitir el conocimiento y en extender sus límites, más que en propugnarlos).

\section{La didáctica de las ciencias complica las cosas innecesariamente}

Una variante de la idea anterior viene a decir que la didáctica de las ciencias (como disciplina aliada de la psicología y la pedagogía) se mete en profundidades excesivas e innecesarias. Así, una queja frecuente hacia nuestro trabajo es que complicamos las cosas con terminologías raras en las que aparecen palabras que no se entienden. No es extraño encontrar en los periódicos airadas cartas al director escritas por maleados profesores en ejercicio que se quejan de que en los libros y documentos sobre la reforma aparecen expresiones como constructivista, metacognición, epistemológico y otras del mismo jaez. Curiosamente, en estas quejas, el reconocimiento de la ignorancia de ciertos conceptos relacionados con la actividad profesional propia se transforma en una acusación hacia el que los utiliza (!).

Detrás de estas quejas subyace, de nuevo, la extendida concepción de que los asuntos propios del aprendizaje y del funcionamiento de la mente humana son temas de sentido común, que se pueden explicar en lenguaje cotidiano y que no requieren de mayores complicaciones 
porque cualquiera (o casi) puede dar su opinión y tener parte de razón. Pocas veces nos paramos a pensar que, independientemente del grado de desarrollo real de una disciplina, una de las primeras tareas a la que deben hacer frente los investigadores consiste precisamente en desarrollar un lenguaje adecuado para describir las observaciones y los marcos conceptuales. Este lenguaje especializado plantea dos tipos de dificultades al lego (tomamos ejemplos propios de la física):

1) El uso de términos específicos, ajenos al lenguaje común (p.e., hamiltoniano, ecuación de onda, vectorial).

2)El uso de términos tomados del lenguaje común, pero con un sentido diferente (p.e., trabajo, energía, campo). El uso de estos términos también causa problemas porque puede confundir al no-especialista.

No cabe duda de que la formalización terminológica contribuye al rigor y se considera necesaria en disciplinas como matemáticas, física o química. Sin embargo, rara vez se considera necesario (ni, por supuesto, conveniente) que los que nos dedicamos al desarrollo de disciplinas blandas emprendamos la tarea de llamar a las cosas por su nombre. Una opinión implícita y generalizada es que haríamos mejor en seguir utilizando términos comunes, accesibles a todos. Así, se llega a la paradoja de que a nadie extraña que los médicos utilicen una terminología propia para describir el funcionamiento (y las enfermedades) del cuerpo humano, mientras que el uso de conceptos específicos para describir el funcionamiento (y las enfermedades) del sistema cognitivo humano por parte de psicólogos, pedagogos y especialistas en didáctica se mira con malos ojos.

\section{Los resultados de las investigaciones didácticas son obvios}

Un profesor universitario de física o química que explore por primera vez a alguna revista de didáctica de las ciencias y lea un artículo de investigación puede quedar decepcionado. Mi experiencia en la formación inicial de profesores confirma la afirmación anterior. Estos futuros profesionales comentan, a veces, que los resultados de muchas investigaciones son obvios, en el sentido de que hubieran sido fácilmente predecibles sin necesidad de realizar ningún estudio ni trabajo de investigación. De hecho, una de las acusaciones frecuentes que debemos encarar en nuestra área es que estamos continuamente redescubriendo cosas evidentes. ¿Qué podemos alegar en nuestra defensa?

Dos maestros enseñan a leer a sus alumnos. Uno de ellos llama cada día a cada uno de sus pupilos según la lista de clase y controla sus avances y dificultades. El otro maestro los llama diariamente de forma aleatoria y controla así sus avances y dificultades. Se comprueba que el método seguido por el primer maestro da lugar a mejores resultados. Indíquese si hubiera sido capaz de predecir el resultado de esta investigación sin necesidad de realizarla y busque una explicación a dichos resultados.
Wong (Gage, 1991) pidió a varios grupos de sujetos que realizasen una prueba similar en la que había dos versiones: una siguiendo el modelo anterior y otra en la cual se indicaba que con el método seguido por el segundo maestro se obtenían mejores resultados (justo lo contrario). Cada sujeto leía únicamente una de las versiones. Realizaron la prueba cuatro grupos de sujetos: estudiantes de ingeniería, estudiantes de psicología, maestros en formación y maestros con experiencia. Los resultados son claros: los sujetos tendían a calificar como obvios los supuestos resultados de la prueba, fueran éstos los que fueran. Los maestros con experiencia no fueron más hábiles que los demás sujetos (por ejemplo, no calificaron como más obvios los resultados reales que los resultados ficticios).

Similares conclusiones se han obtenido en otros campos en los que todos creemos tener derecho a entender y opinar, como son la sociología y la psicología. Parece ser que, cuando un terreno es aparentemente familiar, uno tiende a considerar obvio cualquier resultado o conclusión, independientemente de que sean los reales o vayan justamente en sentido contrario de los reales (!).

Éste es otro problema adicional al que tienen que hacer frente los investigadores en áreas como didáctica de las ciencias cuando pretenden presentar tesis doctorales. Vistas las cosas desde fuera, muchas veces parece que el trabajo realizado no vale la pena ni el investigador merece el título de doctor porque los resultados de su tesis eran obvios.

El conocimiento científico es más fiable que el conocimiento en didáctica de las ciencias y otras áreas «blandas» porque existe y se aplica un «método científico»

Si tuviésemos que comparar nuestra cultura actual con otras anteriores, probablemente una de las diferencias más notables sería el papel que en ella desempeñan la ciencia y la tecnología. El conocimiento científico ha alcanzado un grado de desarrollo sin precedentes y la incidencia de la ciencia y de la tecnología en nuestras vidas cotidianas es de sobra conocida. El conocimiento científico ha pasado a ser el paradigma de conocimiento pleno de rigor, fiabilidad y exactitud, tanto es así que sirve incluso como modelo para otras disciplinas que pugnan por añadir el adjetivo científico a sus métodos y conclusiones. Muchos profesores de ciencias e incluso investigadores en activo creen que estas diferencias cualitativas entre el conocimiento científico y otros tipos de conocimiento se deben a que, en las ciencias experimentales, se utiliza un llamado método científico que se describe en muchos libros como un conjunto ordenado y rígido de pasos más o menos así:

1) Observación desprovista de toda idea o expectativa previa.

2) Detección de una regularidad o patrón.

3) Formulación de una hipótesis o ley general. 
4) Obtención de deducciones contrastables a partir de la hipótesis o ley general.

5) Realización de un experimento de cuyo resultado depende la aceptación de la hipótesis o ley general.

Sin embargo, no está tan claro que la razón por la que el conocimiento científico sea superior a otros tipos de conocimientos es porque se utilice dicho método científico. Se pueden aportar las argumentaciones siguientes:

1) Los filósofos de la ciencia parecen estar de acuerdo en que la actividad de los científicos rara vez puede describirse por la rígida versión anterior del llamado método científico (Chalmers, 1982).

2) Si el problema de las ciencias blandas fuese, fundamentalmente, de tipo metodológico, bastaría con exportar los métodos de la física o la química a dichas disciplinas para que se produjese un avance espectacular.

3) Otras evidencias indirectas indican, además, que la atención que se presta en las disciplinas blandas a las cuestiones metodológicas puede ser tanta o más que la que se presta a dichas cuestiones en las ciencias duras. Así, por ejemplo, según Winston y Blais (1996), comparados con los libros de psicología, sociología y biología, los libros de física general son los que menos uso hacen de los términos variable dependiente y variable independiente. De manera similar, muy pocos libros de física definen experimento como una manipulación o como una observación controlada.

\section{La didáctica es un terreno de investigación «fácil»}

Los que trabajan en áreas de investigación duras tienden a considerar el resto de las disciplinas como un terreno fácil, en el que no existen complicaciones derivadas de las matemáticas y en el que casi cualquiera puede entender y participar. Sin negar la evidencia de que determinados dominios requieren unas capacidades notables de abstracción o de habilidad en el uso de las matemáticas y otros instrumentos conceptuales, es interesante hacer notar lo siguiente:

1) El objeto de estudio de la psicología y de la ciencia cognitiva es el cerebro humano, tal vez el sistema de procesamiento de información más complejo que conocemos. A diferencia de un átomo o de un gas, nuestro objeto de estudio (todavía) se resiste a ser descrito mediante una ecuación (ni de onda, ni de estado).

2) Al contrario que en otras disciplinas, el soporte que ofrecen las matemáticas en nuestro campo es débil. Por ejemplo, los primeros modelos matemáticos para simular la comprensión de textos son relativamente recientes (Kintsch, 1998) y casi siempre la única rama de las matemáticas que se puede aplicar en nuestro dominio es la estadística. No cabe duda de que la aplicación del formalismo matemático haría posible un aumento en el rigor y la calidad de la investigación (aunque, obviamente, requeriría un esfuerzo de aprendizaje de dicho formalismo).
3) A diferencia de las disciplinas duras, las disciplinas blandas se caracterizan por la coexistencia (pacífica o no) de distintos paradigmas o grandes núcleos conceptuales que orientan y dirigen la investigación. En física, por ejemplo, un rango grande de fenómenos (p.e., la mecánica) se estudia y explica mediante un único marco conceptual (las leyes de Newton). En las disciplinas blandas, distintas teorías o paradigmas intentan explicar un mismo rango de fenómenos, sin que necesariamente exista comunicación entre escuelas.

4) En las disciplinas duras, las variables y los conceptos asociados a magnitudes (p.e., velocidad, aceleración) se pueden definir con precisión (aunque después resulte difícil medirlos). En las disciplinas blandas, muchas veces ni siquiera están claros los conceptos o las variables que queremos medir. No es raro, pues, que para clasificar los resultados de una prueba se recurra a dos jueces independientes que aplican criterios difusos, difíciles de explicitar y formalizar.

Los factores anteriores contribuyen, sin duda, a complicar la investigación en nuestra área, no porque se requieran difíciles aparatos matemáticos o conceptuales, sino, justamente, por la ausencia de dichos aparatos articulados con un grado suficiente de solidez.

\section{Si se compara con áreas como física o química, resulta muy fácil publicar en revistas de didáctica de las ciencias}

Para muchos, la supuesta facilidad con la que se realizan investigaciones en psicología y didáctica va acompañada de una similar facilidad para publicar cualquier cosa en cualquier revista de estas áreas. Lo cierto es que publicar en revistas de calidad en las áreas de ciencias sociales es más difícil que publicar en revistas de calidad de áreas de ciencias experimentales o naturales (otro tipo de comparación sería, obviamente, inadecuado). Existen diversos indicadores que avalan la afirmación anterior:

1)Los porcentajes de artículos rechazados en revistas internacionales en áreas como psicología educativa y similares son realmente altos (Rotton y Levitt, 1993), mucho más que los correspondientes a las llamadas ciencias duras como física, química, etc. (Anderson, 1988). Así, por ejemplo, en el estudio de Anderson, el porcentaje medio de artículos aceptados en física es del $61,5 \%$, mientras en psicología es del 18,8\%. Otro ejemplo, según un editorial publicado en la revista Journal of Research in Science Teaching, menos de la mitad de la mitad de los trabajos enviados llegan a ser considerados para su publicación (Good, 1993). Los elevados porcentajes de rechazo en esta revista (por encima del 50\%) se analizan en un comentario publicado por Wandersee y Demastes (1992).

2) Como es sabido, las revistas académicas de más impacto se recogen en las bases de datos conocidas como Science Citation Index y Social Sciences Citation Index 
(puede conseguirse una lista completa en la dirección http://www.isinet.com). Pues bien, según un estudio reciente, el número de contribuciones españolas entre 1988 y 1997 publicadas en revistas recogidas en el Social Science Citation Index (SSCI), en todas las áreas de educación, es realmente bajo (Fernández-Cano, 1999). Los autores con más trabajos publicados contaban con cuatro artículos en revistas SSCI. Esta cifra es evidentemente menor que la que suele ser común en las áreas de ciencias duras, prueba evidente de que las áreas de investigación relacionadas con la educación no han alcanzado todavía en España el nivel suficiente para competir en condiciones de igualdad con la comunidad internacional. Es interesante destacar que, en el estudio citado, una parte significativa de los autores más productivos pertenecen al área de didáctica de las ciencias experimentales.

3) Durante los primeros seis años de evaluaciones de la actividad investigadora, la comisión nacional encargada de la tarea concedió un 54,9\% de solicitudes de tramos en el campo 7 (ciencias sociales, políticas, del comportamiento y de la educación) mientras que en los campos 1 (matemáticas y física) y 2 (química) se concedieron un $64,3 \%$ y un 68,3\% respectivamente (Comisión Nacional Evaluadora de la Actividad Investigadora, 1996).

\section{LOS ALUMNOS Y LA METODOLOGÍA DOCENTE}

Los alumnos son los beneficiarios del servicio público que es la educación en la cual estamos empeñados. Su formación es nuestro objetivo. En el transcurso de la interacción mutua, los profesores desarrollan ideas, creencias y actitudes hacia ellos que afectan a su actuación en el aula. No cabe duda de que la mayor parte de los profesores universitarios de ciencias hacen lo que creen que es mejor para sus alumnos. Su repertorio docente tiene origen, en general, en las vivencias propias cuando él mismo fue estudiante, en las conversaciones e intercambios de opiniones con otros colegas y, en mucha menor medida, en procesos más o menos rigurosos de formación didáctica. El repertorio de actuaciones tolerables para un profesor se complementa con algunas ideas y concepciones generales acerca de lo que es útil, posible y permisible en una clase de universidad. Estos puntos de vista generales modulan y orientan sus concepciones docentes y el posible papel que podría tener la formación didáctica en su trabajo diario. A continuación analizamos algunas de estas concepciones

\section{Las capacidades de los alumnos siempre se distribuyen siguiendo una curva normal}

Una creencia común entre el profesorado universitario de ciencias es que las capacidades de sus alumnos y, por tanto, los resultados de las pruebas que se utilizan para medirlas, se distribuyen siguiendo la conocida distribución gaussiana (Gil, Carrascosa, Furió y MartínezTorregrosa, 1991, p. 96). Esta creencia no sólo es propia de los profesores: muchos investigadores suponen ingenuamente que las medidas relacionadas con las capacidades de los sujetos que intervienen en las investigaciones psicopedagógicas se distribuyen siguiendo una curva normal y basan sus análisis estadísticos (p.e., los análisis de varianza) en esta suposición.

Lo cierto es que existe una amplia evidencia que demuestra que los resultados obtenidos en muchas pruebas psicométricas no siguen una distribución normal. Así, por ejemplo, ya en 1989, Micceri revisó 440 medidas psicométricas o de rendimiento académico con el objetivo de determinar en qué proporción de estas pruebas se observaban distribuciones normales (Micceri, 1989). Como ejemplos cabe citar las pruebas estandarizadas de rendimiento en diversas materias que se utilizan de manera generalizada en Estados Unidos o los resultados obtenidos en pruebas utilizadas en trabajos publicados en revistas especializadas. Aplicando el test de Kolmogovor-Smirnov se encontró que, en el $100 \%$ de los casos, las distribuciones se desviaban significativamente de la curva normal. Sólo en 16 medidas de capacidad los resultados fueron razonablemente próximos a una curva gaussiana (Micceri, 1989, p. 161). No resulta sorprendente que Micceri titulase el artículo en el que informaba de estos resultados como El unicornio, la curva normal y otras criaturas improbables.

La realidad anterior ha motivado que en los últimos años haya surgido una preocupación cada vez mayor por la disponibilidad y el uso de pruebas estadísticas robustas, es decir, pruebas cuyos resultados sean válidos incluso aunque no se cumplan las suposiciones típicas sobre normalidad de las variables que fundamentan las pruebas estadísticas tradicionales.

La creencia en la supuesta normalidad de las capacidades de los alumnos podría ayudarnos a entender determinados comportamientos de los profesores en la evaluación y la calificación. Si un profesor espera que las calificaciones de los alumnos se distribuyan siguiendo una curva normal, es muy probable que consiga algo parecido (calificación según la norma). Por ejemplo, ¿sería esperable que, de manera repetida, un profesor universitario conceda una calificación de sobresaliente a un porcentaje elevado de alumnos aunque éstos superen satisfactoriamente sus exámenes?

Este tipo de creencias en la omnipresencia de la distribución gaussiana (tan común en el mundo inanimado) podría ayudar a entender algunos de los prejuicios e ideas acerca de la inutilidad de algunas intervenciones didácticas: ¿para qué nos vamos a esforzar en mejorar la enseñanza si es normal que los alumnos se distribuyan siguiendo una curva normal?

\section{Cada maestrillo tiene su librillo} La enseñanza no es un trabajo automático ni el maestro un
eco de pensamientos ajenos. El catedrático merecedor de
serlo tiene un sistema y método suyos y, cuando se le 
imponen, pierde su espontaneidad, y sus lecciones son una mezcla extraña de ideas y formas heterogéneas sin unidad ni concierto.

El fragmento anterior sirvió de justificación en 1868 al entonces ministro de Fomento (el radical Manuel Ruiz Zorrilla) para decretar la libertad total del profesor en la elección de métodos y libros de texto y en programa (González y Zaragoza, 1985). La argumentación anterior no es sino una versión algo sofisticada del bien conocido refrán que encabeza este apartado. Este terrible refrán se utiliza como una especie de patente de corso que permite al profesor hacer prácticamente lo que quiera porque, supuestamente, la enseñanza es así y debe ser así. No hay que confundir la libertad de cátedra con la idea que subyace al refrán anterior. En un caso se trata de un tema legal y constitucional y en el otro nos referimos a la realidad de la práctica docente.

A la vista de una convicción tan libertaria y tan arraigada en el profesorado, cabría esperar una amplia variedad de técnicas docentes y de enfoques didácticos en nuestras universidades. Evidentemente, no es esta la situación real, como se explica en el siguiente apartado.

\section{La masificación es la causa que impide desarrollar metodologías docentes más activas}

Cuando se discute, con profesores universitarios de ciencias, la necesidad de utilizar estrategias docentes más activas, siempre acabamos igual: «Sí, sí, la teoría es muy bonita, pero con 100 alumnos en clase ya me dirás qué se puede hacer.» La idea que subyace tras este lamento es: los profesores disponen de un repertorio variado de estrategias docentes que obrarían maravillas en el aprendizaje. Sin embargo, el contexto no les deja aplicar este rico repertorio de alternativas. ¿Es esto cierto? Evidentemente, no. Pocos profesores de universidad son capaces de sugerir muchos cambios realmente originales en sus actividades docentes si tuviesen solamente 15 alumnos en una asignatura.

La realidad anterior resulta interesante por varios motivos. En primer lugar, pone en evidencia que el repertorio docente de los profesores es realmente limitado (Plan Nacional de Evaluación de la Calidad de las Universidades, 2001). Las propuestas que surgen como resultado de dicha actividad se limitan casi siempre a constatar que los alumnos estarían mejor atendidos y que, tal vez, podrían realizar trabajos (pero con cuidado, no vaya a ser que copien). En segundo lugar, cuando se profundiza un poco más, se comprueba que las alternativas que sugieren los propios profesores están, según ellos, llenas de problemas e inconvenientes (amén de que exigen más trabajo por su parte) de forma que la clase magistral acaba siendo no sólo el único método docente posible, sino el único deseable. Incluso en aquellas asignaturas optativas con un número reducido de alumnos la clase magistral (dura o suavizada) es lo único que aciertan a organizar muchos profesores.

Esta bondad a ultranza de la clase magistral se pone todavía más de manifiesto cuando se eliminan, de los cambios propuestos en la actividad docente que resultarían si el profesor tuviera menos alumnos por clase, todo aquello que se refiera a cambios cuyo origen estuviera en el alumno (p.e., sería más fácil que éstos preguntaran dudas). Este análisis detallado demuestra que la mayor parte de los cambios que se producirían en la enseñanza tienen que ver con cosas que harían los alumnos, más que los propios profesores. Curiosamente, la respuesta de la mayor parte de los profesores de ciencias a las evidencias anteriores no es «necesito aprender más técnicas docentes» sino una derivación del tema de conversación a las condiciones generales de trabajo y de salario con las que tienen que apechugar y a lo injusto del sistema de promoción en la universidad.

\section{CONCLUSIONES Y PERSPECTIVAS}

La relación anterior no agota el posible repertorio de puntos de vista inadecuados sobre la didáctica de las ciencias, tan comunes en el profesorado universitario de ciencias. Sin embargo, constituyen un punto de partida aceptable para plantear algunas de las necesidades y carencias en su formación. Una vez planteados, analizados y cuestionados los puntos de vista inadecuados, tal vez resulte más fácil para algunos profesores darse cuenta de que, tal como sucede en ciencias, algunas ideas y evidencias de sentido común no sirven para entender la realidad, causan problemas en la actuación diaria y deberían llevarnos a propugnar, también en nuestra labor docente, un proceso de cuestionamiento de lo evidente, de lo aceptado por todos y de lo nunca puesto en tela de juicio (Gil y Vilches, 1999) (sana práctica que está en la base del avance científico).

La buena noticia es que la didáctica de las ciencias puede ofrecer algún apoyo a la renovación y mejora de la labor docente del profesor desde los necesarios procesos de formación. Ya existen algunos tímidos intentos de formación del profesorado universitario que se han desarrollado en algunas universidades con un éxito diverso. La mala noticia es que todavía no estamos en condiciones de ofrecer soluciones milagrosas fuera de toda duda para los problemas de aprendizaje y enseñanza de las ciencias. Pero podemos ofrecer orientaciones y propuestas de trabajo y de actuación que vayan más allá que el dictado de apuntes. Incluso podemos ofrecer algunas sugerencias que funcionan en clase masificadas (Campanario, 2002b). Sin embargo, no cabe esperar de nosotros una receta que garantice siempre el éxito en la docencia de las ciencias en la universidad. Además, la investigación en didáctica de las ciencias se ha orientado tradicionalmente a los niveles de enseñanza secundaria. Todavía hay mucho que explorar y aprender sobre los problemas de enseñanza y aprendizaje de las ciencias en el nivel superior. 
Creemos que la enseñanza no es una tarea fácil (quizá sea la actividad humana más difícil), ni algo que pueda realizar bien simplemente cualquiera que sepa su asignatura o que investigue con éxito en un terreno especializado como, por ejemplo, la química de polímeros o la física del estado sólido. Al igual que en cualquier otra actividad profesional, la formación específica suele ser imprescindible y será positiva tanto para el profesional como para el beneficiario del servicio público al que se atiende. Esta línea de pensamiento y actuación no se diferencia mucho de la tendencia en el resto de las profesiones. Cada vez aumentan más los requisitos de formación que se piden para el desempeño de cualquier trabajo medianamente cualificado. La formación psicopedagógica de los profesores de Universidad es una necesidad evidente para la que no existe todavía deman$d a$. Como se ha indicado más arriba, quizá el primer paso necesario para conseguir esta demanda sea convencer a los propios profesores universitarios de ciencias de que un mínimo de formación psicopedagógica puede ayudarles a realizar mejor su trabajo. Cualquier sugerencia en este sentido será bien recibida.

\section{REFERENCIAS BIBLIOGRÁFICAS}

ANDERSON, G.C. (1988). Getting science papers published: where it's easy, were it's not. The Scientist, 19, pp. 26-27.

CAMPANARIO, J.M. (1998). ¿Quienes son, qué piensan y qué saben los futuros maestros y profesores de ciencias?: Una revisión de estudios recientes. Revista Interuniversitaria de Formación del Profesorado, 33, pp. 121-140 (http:// www.uah.es/otrosweb/jmc)

CAMPANARIO, J.M. (2002a). Asalto al castillo: ¿A qué esperamos para abordar en serio la formación didáctica de los profesores universitarios de ciencias? Enseñanza de las Ciencias, 20 (2), pp. 315-325 ( http://www.uah.es/otrosweb/ jmc).

CAMPANARIO, J.M. (2002b). ¿Qué puede hacer un profesor como tú con una clase tan masificada como ésta? Docencia Universitaria, 3(1), pp. 28-42 (http://www.uah.es/otrosweb/ jmc).

CAMPANARIO J.M. y MOYA, A. (1999). ¿Cómo enseñar ciencias? Las principales tendencias y propuestas. Enseñanza de las Ciencias, 17 (2), pp. 179-192.

CHALMERS, A.F. (1982). ¿Qué es esa cosa llamada ciencia? Madrid: Siglo XXI Editores.

COMISIÓN NACIONAL EVALUADORA DE LA ACTIVIDAD INVESTIGADORA (1996). Seis años de evaluaciones. Madrid: MEC.

FERNÁNDEZ-CANO, A. (1999). Producción educativa española en el Social Sciences Citation Index (1988-1997). Revista Española de Pedagogía, 57(214), pp. 509-524.

GAGE, N.L. (1991). The obviousness of social and educational research results. Educational Researcher, 20(1), pp. 10-16.

GIBSS, W.W. y FOX, D. (2000). Enseñanza de las ciencias. Investigación y Ciencia, pp. 77-81.

GIL, D., CARRASCOSA, J., FURIÓ, C. y MARTÍNEZTORREGROSA, J. (1991). La enseñanza de las ciencias en la educación secundaria. Barcelona: ICE. Universitat de Barcelona.
GIL, D. y VILCHES, A. (1999). Problemas de la educación científica en la enseñanza secundaria y la universidad: contra las evidencias. Revista Española de Física, 13(5), pp. 10-15.

GONZÁLEZ, I. y ZARAGOZA, G. (1985) Siglo y medio de libros de texto. Cuadernos de Pedagogía, 122, pp. 4-6.

GOOD, R. (1993). Editorial: The JRST editorial process. Journal of Research in Science Teaching, 30(3), pp. 219-221.

KINTSCH, W.(1998). Comprehension: A paradigmfor cognition. Cambridge: Cambridge University Press.

MELLADO, V. (1996). Concepciones y prácticas de aula de profesores de ciencias en formación inicial de primaria y secundaria. Enseñanza de las Ciencias, 14(3), pp. 289-302.

MICCERI, T. (1989). The Unicorn, the normal curve and other improbable creatures. Psychological Bulletin, 105(1), pp. 156-166.

PLAN NACIONAL DE EVALUACIÓN DE LA CALIDAD DE LAS UNIVERSIDADES (2001). Licenciado en química. Autoinforme de evaluación. Alcalá de Henares: Universidad de Alcalá de Henares.

PORLÁN, R., RIVERO, A. y MARTÍN, R. (1997a). Conocimiento profesional y epistemología de los profesores I: Teoría, métodos e instrumentos. Enseñanza de las Ciencias, 15(2), pp. $155-171$.

PORLÁN, R., RIVERO, A. y MARTÍN, R. (1997b). Conocimiento profesional y epistemología de los profesores II: Estudios empíricos y conclusiones. Enseñanza de las Ciencias, 16(2), pp. 271-288.

ROTTON, J. y LEVITT, M. (1993). Citation impact, rejection rates and journal value. American Psychologist, 48(8), pp. 911-912.

WANDERSEE, J.H. y DEMASTES, S. (1993). An analysis of the relative success of qualitative and quantitative manuscripts submitted to the Journal of Research in Science Teaching, 29(9), pp. 1005-1010.

WINSTON, A.S. y BLAIS, D.J. (1996). What counts as an experiment?: A transdisciplinary analysis of textbooks, 19301970. American Journal of Psychology, 109, pp. 599-616. 